\title{
Cooperation Between Japan's NIID and China CDC in Japan's COVID-19 Response
}

\author{
Takaji Wakita ${ }^{1, *}$
}

Emerging infectious diseases such as avian influenza and coronavirus disease 2019 (COVID-19) are important issues for health security in every country. Furthermore, since no country borders exist for these emerging infectious pathogens, international cooperation is indispensable to reduce disease transmission. For this reason, the National Institute of Infectious Diseases, Japan (NIID) reached an agreement with China CDC on a cooperative framework for infectious disease research and signed a memorandum of understanding in August 2006. Based on this memorandum, the NIID has further deepened cooperation between the two organizations and has been promoting research cooperation and the exchange of human resources for infectious disease control.

The NIID and China CDC have a history of information sharing and research cooperation between individual researchers and laboratories. In particular, the NIID provided considerable support to China for the World Health Organization (WHO) polio eradication program. Based on this relationship, the NIID has been organizing to deepen the cooperative relationship with China $\mathrm{CDC}$, which is the core to infectious disease research and response in China. Furthermore, in April 2006, a similar memorandum of cooperation was signed with the Korea Centers for Disease Control and Prevention [now the Korea Disease Control and Prevention Agency (KDCA)]. Based on the cooperative relationship between these three countries, attempts are being made to strengthen cooperation in infectious disease control in East Asia. Since 2006, the Japan-China-Korea Forum on Communicable Disease Control and Prevention has been held with the NIID, China CDC, and KDCA. In this forum, information is exchanged on emerging infectious diseases including new strains of influenza and severe fever with thrombocytopenia syndrome, as well as core activities such as surveillance and field epidemiology training programs.

The present COVID-19 pandemic offers a stark reminder of the importance of preparedness for emerging and reemerging infectious diseases as the world continues to struggle with COVID-19. At the end of 2019, cases of pneumonia of unknown cause (PUE) were reported in Wuhan, China. Initially, there were reports of an outbreak that occurred among people in relation to the Huanan Seafood Wholesale Market in Wuhan, and the epidemic then began to spread worldwide. On January 10, 2020, the viral genome sequence of COVID-19 virus was reported, and the NIID immediately started to develop a viral genome polymerase chain reaction (PCR) test. On January 15, the first domestic case of COVID-19 was confirmed in Japan. A patient with pneumonia stayed in Wuhan and was reported to the WHO based on International Health Regulations (1).

On January 30, the WHO Emergency Committee declared COVID-19 a Public Health Emergency of International Concern (2). After that, the Diamond Princess cruise ship, which arrived at Yokohama Port on February 3, was quarantined for 2 weeks starting from February 5. In total, 712 of 3,711 crew members and passengers tested positive for COVID-19 (331 of whom were asymptomatic pathogen carriers), and 13 deaths were confirmed. All passengers were subsequently required to self-quarantine at home for an additional 14 days after disembarking, and as a result, the infection did not spread from the cruise ship to Japan (3-5).

Due to its spread and severity, on March 11, the WHO declared the COVID-19 outbreak a global pandemic. In Japan, the number of imported cases from foreign countries increased from the beginning of March, and the number of cases without a clear infectious source continued to increase in the middle of March. Furthermore, in late March, the number of infection clusters were increasing rapidly, mainly in urban areas, and a sharp increase of newly infected cases was seen. In response to this situation, on March 10, the Japan Cabinet made the decision to revise in part and activate the Act on Special Measures for Pandemic Influenza and New Infectious Diseases Preparedness and Response to include and stipulate COVID-19. On March 28, "Basic Action Policies for 
Countermeasures Against the Novel Coronavirus Disease" were announced, and it was considered important to reduce the number of infected cases and maintain the medical care provision system and social functions. Avoiding the "Three Cs" (i.e., closed spaces with poor ventilation, crowds, and close-contact settings) and containing outbreaks and clusters through active epidemiological studies were therefore promoted.

On April 7, due to the rapid increase in new cases and the tight medical care provision system, a state of emergency was declared for 7 prefectures in Japan. Citizens of these prefectures were told to reduce contact by $80 \%$ and to refrain from going out and traveling across prefectural borders, telework was promoted, and some businesses were shut down. The state of emergency was expanded to all prefectures on April 16. As the epidemic spread from March to April, the day with the highest number of newly reported cases in the National Epidemiological Surveillance of Infectious Disease system was April 9, but the most frequent date of symptom onset was April 1. It was therefore considered that the number of infected cases rose sharply from mid-March, peaked in early April, and then decreased and began to flatten in mid-May. The state of emergency was gradually lifted from May 14 to 25 , when it was officially lifted in all prefectures (6). After easing restrictions against going out and using facilities, the level of socioeconomic activity gradually increased, and on June 19, the restriction on traveling across prefectural borders was completely lifted nationwide. However, since mid-June, the number of new cases started to increase again, mainly among those aged in their 20s and 30s and in large urban areas and their surrounding vicinities (7).

Since June, the spread of infection among young people has been remarkable, especially in connection with restaurants with entertainment venues in the Shinjuku area. However, as the number of infected cases increases among the younger population, the number of middle-aged and older people who are at high risk of becoming severely infected is also increasing. As of July 22, 18 severely ill patients required ventilator support in Tokyo, and this number has been rising.

After the state of emergency was lifted, Japan's major countermeasures against COVID-19 included basic individual infection prevention (e.g., wearing masks, hand hygiene, social distancing), avoiding the "Three Cs", and cluster surveillance in active epidemiological surveys with a focus on securing an effective medical care provision system. However, after lifting the restrictions on going out, doing business, and traveling, the number of infected cases surged again. The risk of infection is considered to be high in situations in which a person talks in a loud voice or exhales in a "Three Cs" setting. Cluster infections originating from facilities such as pubs and daytime karaoke bars were detected. In addition, infections often spread to medical institutions and welfare facilities for the aged. At present, balance is needed between socioeconomics and infection control to substantially reduce the risk of infection in places and industries where such risk is considered high. In addition, older people at high risk of severe disease and other generations with whom they may come into contact also need to take extra steps to reduce the risk of infection.

Currently, non-pharmaceutical interventions are the main focus, but the rapid development of vaccines and therapeutic agents is needed before a fundamental solution for COVID-19 is possible. In addition, although the seasonality of the pandemic remains unclear, an urgent response is needed during influenza season. It is therefore necessary to combine wisdom from the fields of clinical medicine, infectious diseases, public health, and virology. In that sense, cooperation between the NIID, China CDC, and KDCA is the most important factor.

doi: $10.46234 / \mathrm{ccdcw} 2020.234$

\# Corresponding author: Takaji Wakita, wakita@nih.go.jp.

${ }^{1}$ National Institute of Infectious Diseases, Tokyo, Japan.

Submitted: October 28, 2020; Accepted: November 05, 2020

\section{REFERENCES}

1. National Institute of Infectious Diseases. Infectious agents surveillance report (IASR): Outbreak of community-acquired infection in Wuhan, China, which was detected in the wake of the first case of COVID-19 infection in Japan. IASR 2020: Vol. 41: 143 - 144. https://www.niid. go.jp/niid/ja/diseases/ka/corona-virus/2019-ncov/2488-idsc/iasr-news/ 9729-485p04.html. [2020-10-21].

2. National Institute of Infectious Diseases. Infectious diseases weekly report(IDWR), Japan: COVID-19 infection. (as of the week 7 of 2020). https:/www.niid.go.jp/niid/ja/diseases/ka/corona-virus/2019-ncov/2487idsc/idwr-topic/9446-idwrc-2007.html. [2020-10-21]. (In Japanese).

3. Yamagishi T, Kamiya H, Kakimoto K, Suzuki M, Wakita T. Descriptive study of COVID-19 outbreak among passengers and crew on Diamond Princess cruise ship, Yokohama Port, Japan, 20 January to 9 February 2020. Euro Surveill 2020;25(23):2000272. http://dx.doi.org/10.2807/ 1560-7917.ES.2020.25.23.2000272.

4. Sekizuka T, Itokawa K, Kageyama T, Saito S, Takayama I, Asanuma H, et al. Haplotype networks of SARS-CoV-2 infections in the Diamond Princess cruise ship outbreak. Proc Natl Acad Sci USA 2020;117(33): 20198 - 201. http://dx.doi.org/10.1073/pnas.2006824117.

5. Kakimoto K, Kamiya H, Yamagishi T, Matsui T, Suzuki M, Wakita T. 
Initial investigation of transmission of COVID-19 among crew members during quarantine of a cruise ship - Yokohama, Japan, February 2020. MMWR Morb Mortal Wkly Rep 2020;69(11):312 - 3. http://dx.doi.org/ 10.15585/mmwr.mm6911e2.

6. National Institute of Infectious Diseases. Infectious diseases weekly report, Japan: Current situation of COVID-19 infection in Japan (as of the week 27 of 2020). https://www.niid.go.jp/niid/ja/diseases/ka/corona- virus/2019-ncov/2487-idsc/idwr-topic/9746-idwrc-2027.html. [202010-21). (In Japanese).

7. National Institute of Infectious Diseases. Infectious diseases weekly report, Japan: Current situation of COVID-19 infection in Japan (as of the week 30 of 2020). https://www.niid.go.jp/niid/ja/diseases/ka/coronavirus/2019-ncov/2487-idsc/idwr-topic/9794-idwrc-2030.html. [202010-21]. (In Japanese).

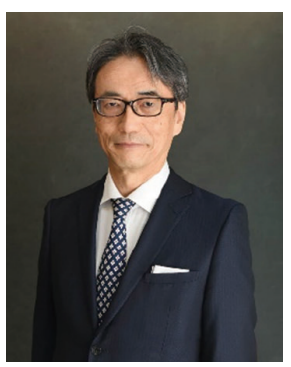

Takaji Wakita, MD, PhD

Director-General, National Institute of Infectious Diseases, Japan 